\title{
Response to Fall 2011 Article: Clinical Molecular Testing: Subspecialty, Entry-level or Specialist Certification
}

It is easy to see the explosion of molecular tests and methods now being employed to improve the diagnostic information provided by the laboratory to clinicians in order to benefit a patient. And, it is true that not all molecular laboratories are the same when it comes to test menu, instrumentation or methods.

The ASCP Board of Certification is proud to sponsor the Molecular Biology certification exam because we believe certification matters in the laboratory and for the health of the patient. When due diligence was conducted with the former NCA staff for the two molecular exams that existed pre-merger, it was determined both exams were entry level and the use of the word "specialty" for the former NCA exam did not contain a different body of knowledge. NCA used the word specialty to convey a unique area of the laboratory, like hematology or cytogenetics, whereas the BOC uses it denote a more complex body of knowledge requiring either two or three years of work experience, academic degree and/or certification for that content area. (Please refer to our website: www.ascp.org/BOC for information on certification) For this reason the two

Sincerely,

Walter Oliveira, BS, MLS(ASCP) ${ }^{\mathrm{CM}} \mathrm{SI}$

Chair, Board of Governors

ASCP Board of Certification exams were merged into one, entry level certification.

The MB exam committee is comprised of content experts and practicing technologists, directors, educators and pathologists who collectively know the field as well as the need for competent and qualified staff. Because the current $\mathrm{MB}$ exam is meant to be an entry level certification, the eligibility routes are appropriate. We have an established process that welcomes collaboration with other organizations when a request has been made to explore the need for a new certification exam, entry level or specialty. The amount of resources required for this endeavor are extensive but when we work together it is rewarding for the organizations, the applicant, the employer and the patient. There may be a need for a true specialty level MB exam and if developed it would align with our other specialty exams and have a three year work requirement and or graduate degree for eligibility.

We believe certification matters and a prospective employee with $\mathrm{MB}(\mathrm{ASCP})^{\mathrm{CM}}$ will have a foundation to build upon when hired. 